\title{
Genre, métissage et transactions coloniales aux Indes néerlandaises (1900-1942)
}

Gender, Métissage and Colonial Engagements: The Case of the Dutch East Indies (1900-1942)

\section{Frances Gouda}

Traducteur : Florence Cabaret

\section{(2) OpenEdition Journals}

Édition électronique

URL : https://journals.openedition.org/clio/9995

DOI : 10.4000/clio.9995

ISSN : 1777-5299

Éditeur

Belin

Édition imprimée

Date de publication : 1 mai 2011

Pagination : 23-44

ISBN : 978-2-8107-0157-5

ISSN : 1252-7017

Référence électronique

Frances Gouda, «Genre, métissage et transactions coloniales aux Indes néerlandaises (1900-1942) 》, Clio. Femmes, Genre, Histoire [En ligne], 33 | 2011, mis en ligne le 01 mai 2013, consulté le 26 avril 2022. URL : http://journals.openedition.org/clio/9995; DOI : https://doi.org/10.4000/clio.9995 


\section{Genre, métissage et transactions coloniales aux Indes néerlandaises $(1900-1942)^{1}$}

Frances GOUDA

[Les Hollandais] ont dépouillé le vieil homme européen et sont entrés, quelques-uns dans la peau, mais tous dans le régime du Javanais [...] Les femmes ont fait mieux. Elles ont laissé là tout l'attirail de la toilette occidentale, corsets, jupons, etc. Toute la journée, de l'extrême matin jusqu'à la chute du jour, elles ont une tenue directement empruntée de l'indigène qui s'appelle sarong kabaaia ${ }^{2}$.

Joseph Chailley-Bert, l'un des fondateurs, en 1893, avec « M. Léon Say et quelque étrangers amis des colonies » de l'Institut colonial international de Paris, se présente souvent comme expert en matière de gouvernement colonial. Directeur de la Quinaine coloniale, il défend avec ardeur l'idée de connaissances comparatives. Ainsi écrit-il dans l'introduction à son ouvrage sur Java et ses habitants:

Les nouveaux venus de la colonisation (et c'est bien ce qu'est la France, dont l'entrée dans la politique coloniale ne date que de moins de vingt ans) ont intérêt à se mettre à l'école de leurs devanciers : Anglais et Hollandais ${ }^{3}$.

Il fait aussi remarquer que ce nouvel ouvrage consacré à la société coloniale de Java, qu'il décrit à juste titre comme le cœur des Indes néerlandaises, constitue la suite logique de ses précédents travaux sur

\footnotetext{
1 Nous tenons à remercier Rebecca Rogers pour son aide précieuse lors de l'édition de cet article.

2 Chailley-Bert $1900: 72$.

3 Chailley-Bert 1900 : vii.
} 
Les Anglais à Hong Kong (1891) et Les Anglais en Birmanie (1892)4. Dans la dédicace, il témoigne d'un respect tout particulier pour l'administration coloniale hollandaise :

Ce livre est dédié respectueusement... à Monsieur J.-D. Fransen van de Putte, le ministre réformateur des colonies hollandaises... par son élève, son admirateur et son $\mathrm{ami}^{5}$.

De fait, Java et ses habitants ne ressemble en rien aux travaux que Joseph Chailley-Bert avait pu commettre sur le colonialisme britannique. En effet, il témoigne d'une attention soutenue pour de nouveaux sujets tels que «la question des métis» et l'attitude désenchantée des femmes blanches devant la fréquence des relations interraciales et l'existence d'enfants métis'.

À partir des observations de Joseph Chailley-Bert, je m'intéresse ici aux relations de genre dans les Indes néerlandaises, où une tradition ancienne de mariage et de concubinage entre individus de couleur différente a produit une hybridité démographique dont j’analyse les ramifications socioculturelles. Ce long passé de métissage a contribué à «normaliser » le phénomène des naissances interraciales à la fin du XIX siècle: de fait, on estime qu'à l'époque où ChailleyBert arpente Java, environ $75 \%$ de la population européenne officiellement recensée dans les Indes hollandaises a des parents de couleurs différentes ${ }^{7}$. L'intégration progressive de l'intimité interraciale et des naissances d'enfants métis fait la particularité de la société coloniale hollandaise, comparée aux relations de genre dans les autres colonies européennes d'Asie (comme dans l'Inde britannique). Or, à cette époque, la réalité du métissage sur le continent sud-asiatique a tendance à être négligée, malgré le nombre important de métis. Le recensement britannique de 1901 indique qu'environ $50 \%$ des adultes britanniques de cette région entrent dans la catégorie des «Eurasiens $»^{8}$. À l'inverse, en Indochine, que Napoléon III commence à occuper dans les années 1860 avant que les

\footnotetext{
4 Chailley-Bert 1891-1892.

5 Chailley-Bert 1900 : page de garde, non numérotée.

6 Chailley-Bert 1900 : vii, 89-96.

7 Ce pourcentage est cité dans Stoler 1992.

8 Marshall 1992 : 192 ; voir aussi Clancy-Smith \& Gouda 1998.
} 
colons ne s'y installent au cours de la décennie suivante, la question du concubinage entre Français et Indochinoises est intégrée au débat politique alors même qu'en 1900, il n'y a que quelques centaines de métis dans ce pays?.

Si, comme l'a bien montré Emmanuelle Saada, le discours colonial n'a pas fait du métissage une priorité de l'Algérie française, en Indochine, le procureur général soulève dès 1897 la question des effets nocifs des pratiques sexuelles interraciales ${ }^{10}$. Et c'est avec « une certaine répugnance » qu'il attire l'attention sur les

funestes conséquences de ces cohabitations irrégulières qui dégradent le magistrat, compromettent son autorité et son prestige et quelquefois - ce qui est pis encore - son honneur.

Il prend ainsi des mesures disciplinaires afin que « la magistrature donne partout en Cochinchine l'exemple de la tenue, de la conduite et de la moralité ». En 1901, le gouverneur général évoque à nouveau les conséquences néfastes du concubinage sur l'honneur et le prestige de la France; il met en garde les fonctionnaires français de toutes les provinces en soulignant

les inconvénients que présente au point de vue de la dignité professionnelle la cohabitation avec des femmes indigènes [...] L'expérience a démontré que l'influence des concubines indigènes est presque toujours funeste à la réputation des fonctionnaires qui les admettent dans l'intimité de leur existence ${ }^{11}$.

Au début du siècle, l'Indochine française n'a que quelques années d'existence. Le fait que seul un petit nombre de fonctionnaires et de colons français y résident laisse accroire que les relations sexuelles interraciales représentent une menace pour l'image que l'on se fait de la supériorité de la culture française et de l'honneur de chacun de ses représentants.

À partir des différences de préoccupations politiques et de traitement des métis en Inde britannique et en Indochine française, j’aborderai un ensemble de points liés aux tensions suscitées par la confrontation des questions de genre, de sexualités et de couleurs

\footnotetext{
9 Pomfret 2009.

10 Saada 2007.

11 Cité par Saada 2003 : 4.
} 
dans l'Indonésie coloniale au moment de l'apogée de l'impérialisme occidental en Asie. La démarche comparative permet en effet de répondre à plusieurs types d'interrogations. Étant donnée l'ancienneté des relations interraciales et des métis à Java, les différences de couleurs sont-elles automatiquement associées à des différences de pouvoir, de hiérarchies sociales et de prestige culturel ? Ou, pour le formuler autrement, la blancheur relative des Européens leur vaut-elle systématiquement pouvoir et respect, comme on l'entend souvent dire? Comment les femmes, tant européennes qu'indigènes, sontelles impliquées dans les pratiques de la «culture hollandaise d'outremer », au cœur de l'archipel indonésien, et quel est le but du nouveau régime de mœurs qui fait son apparition autour de 1900 et cherche à mobiliser les femmes blanches dans un effort concerté de consolidation des clivages entre les Européens et les habitants de ces îles (c'est-à-dire la population indigène) ${ }^{12}$ ? Doit-on y voir une tentative de protéger les profits du capital, d'affermir le contrôle des Hollandais en mettant l'accent sur la réalité politique et juridique, comme l'a fait remarquer récemment Romain Bertrand dans son impressionnante contribution intitulée État colonial, noblesse et nationalisme à Java: "Les Européens étaient considérés comme citoyens néerlandais [et] les Indigènes comme sujets de la Couronne » ?13 Enfin, est-il raisonnable de penser que les effets cumulés d'à peu près trois siècles de métissage issu de relations sexuelles entre colonisateurs blancs et femmes indigènes ont déjoué les efforts «modernes » de 1900, quand les Hollandais commencèrent à encourager l'émergence de nouvelles sensibilités raciales dans les Indes néerlandaises?

\section{Intimité et métissage avant $\mathbf{1 9 0 0}$}

Depuis le XVII e siècle, les communautés coloniales hollandaises implantées en Asie (surtout à Batavia, mais aussi à Macassar, Malacca, Galle ou Colombo) ont mis en place des fonctionnements sociopolitiques où le statut des Européens était intimement lié aux entreprises commerciales locales mais aussi aux routes commerciales

\footnotetext{
12 Voir Gouda 2008 [1995].

13 Bertrand $2005: 594$.
} 
qui sillonnaient l'Asie, ainsi qu'aux réseaux du commerce des esclaves. Dans nombre de régions de l'Asie du Sud et du Sud-Est, la plupart des colons hollandais établirent des relations physiques et matérielles avec les populations indigènes. Pendant les deux premiers siècles de présence hollandaise en Asie, le pouvoir économique et politique était fondé sur l'idée que l'on se faisait des bienfaits de l'éducation hollandaise et de l'apprentissage du hollandais, mais aussi sur les bénéfices économiques que l'énorme infrastructure commerciale que représentait la Compagnie hollandaise des Indes orientales (Verenigde Oostindische Compagnie ou VOC) pouvait apporter. Le statut social et l'autorité politique des Européens dépendaient en grande partie d'affiliations locales régulièrement renforcées ou fragilisées par le jeu des alliances entre familles. Au gré des variations du sort de ces familles d'Asie du Sud-Est, les femmes servaient à tisser des liens sociaux et contribuaient ainsi à la cohésion des élites coloniales locales ${ }^{14}$. Jusqu'à la deuxième moitié du XIX siècle, à Batavia et dans d'autres communautés coloniales néerlandaises en Asie, un mariage bien négocié avec une veuve haut placée ou avec une future héritière (à la peau souvent très foncée) était l'un des meilleurs moyens d'assurer sa réussite financière, sa position sociale et son rayonnement politique ; la blancheur relative de l'Européen en question était alors très secondaire ${ }^{15}$. De plus, du milieu du XVII ${ }^{e}$ siècle jusqu'au début du $\mathrm{XX}^{\mathrm{e}}$ siècle, les Hollandais avaient partout pris l'habitude de vivre en concubinage avec ce que l'on appelait pudiquement une gouvernante indigène (nyai). Pour l'essentiel, ces nyai enfantèrent la population métissée des Indes néerlandaises, de même que "Hagar fut la mère d'Ismaël et de ses descendants $»^{16}$.

Les sources écrites détenues par les communautés et les entreprises hollandaises d'Asie du Sud et du Sud-Est remettent en cause l'idée reçue de sociétés coloniales prioritairement organisées selon des hiérarchies fondées sur la couleur de peau ${ }^{17}$. Si l'on en croit ces clichés, les nouveaux arrivants en provenance d'Europe étaient

\footnotetext{
14 Davidoff \& Hall 1987.

15 Bosma \& Raben 2008.

16 Baay $2008: 222$.

17 Bosma \& Raben 2008 : 59-60.
} 
aussi perçus comme les «seigneurs et maîtres » tout-puissants tandis que les masses indigènes se contentaient de trimer dans les rizières, étaient employées comme esclaves, domestiques ou travaillaient depuis le début du XIX ${ }^{\mathrm{e}}$ siècle pour des salaires misérables dans des plantations de café, de thé, de sucre, de tabac et de caoutchouc puis, plus tard, dans les puits de pétrole ainsi que dans les mines de bauxite. Ces images toutes faites cristallisent un certain nombre de stéréotypes orientalistes rebattus, dont on a encore du mal à se défaire. Parmi d'autres exemples révélateurs, on peut citer le commentaire, au début du XIX ${ }^{e}$ siècle, de l'administrateur colonial et ethnographe britannique John Crawfurd, qui souligne dans son Histoire de l'archipel indien (1820) le contraste entre «le génie viril et généreux des nations européennes» et "la faiblesse, l'incapacité et la puérilité qui ont toujours caractérisé l'Asie $»^{18}$. Étant donnée la prégnance de ce genre de préjugés occidentaux dans l'histoire de l'impérialisme européen en Asie, il y a une certaine ironie à constater aujourd'hui que cette conception de hiérarchies inévitables et étanches fondées sur la couleur de la peau ait persisté ou ait refait surface dans l'historiographie (post)coloniale contemporaine.

$\mathrm{Si}$, à l'inverse, nous nous penchons davantage sur les interactions sociales et sur le rôle économique des soldats et marins européens, mais aussi des métis convertis au christianisme et de la multitude d'esclaves qui occupaient des positions cruciales d'intermédiaires au sein des sociétés coloniales, une tout autre configuration se fait jour. Récemment, dans leur ouvrage intitulé Être hollandais aux Indes: Une histoire de la créolisation et de l'Empire, 1500-1920, les historiens hollandais Ulbe Bosma et Remco Raben ont pu ainsi écrire que «la vision simpliste et attrayante » de sociétés coloniales universellement caractérisées par une hiérarchie raciale particulièrement forte et rigide, fondée sur la supériorité des Blancs et la subordination des populations de couleurs est «fausse », surtout dans le cas des sociétés coloniales hollandaises d'Asie ${ }^{19}$. Si la politique coloniale hollandaise n'a pas cherché, même d'une manière plus ou moins déguisée, à encourager un métissage propice à la constitution d'une réserve de

\footnotetext{
18 Knapman 2008.

19 Bosma \& Raben 2008 : 60.
} 
main-d'œuvre de base où il aurait été facile de puiser, il n'en est pas moins vrai que les réalités quotidiennes de la vie coloniale ont conduit les colonisateurs à reconnaitre que l'hybridité démographique due à leur présence impliquait des formes d'accommodements et d'incorporations ${ }^{20}$. Ainsi, les pères hollandais reconnaissaient-il régulièrement leurs enfants métis si bien qu'à la fin du XIX ${ }^{\mathrm{e}}$ siècle, de nombreux individus issus de relations entre Hollandais et femmes indonésiennes avaient obtenu une reconnaissance officielle de leur statut d'Européens. Parmi eux, quelques-uns ont fondé des familles dont les membres ont la peau mate et possèdent des propriétés et des fortunes considérables. Au début du XXe siècle, certains occupent des fonctions importantes dans le secteur privé, ou commencent à obtenir des postes dans les premiers échelons de la bureaucratie coloniale. Mais, quand ils étaient abandonnés par leur père blanc, il est fort probable que la majorité de ces métis a été élevée par leur mère dans une pauvreté plus ou moins extrême, dans des villages à la campagne ou dans des quartiers situés à la périphérie des villes sans que leur père européen ne leur apporte jamais aucun soutien financier.

Il fait donc peu de doute que la communauté coloniale hollandaise d'Asie a été constituée d'une minorité d'individus blancs aux yeux bleus venus d'Europe du Nord et d'une majorité d'Européens à la peau mate, au « teint basané [avec] une petite couleur de chocolat-aulait $»^{21}$. Ainsi, en 1796, les nouveaux arrivants de Grande-Bretagne furent-ils extrêmement surpris quand ils découvrirent les «bourgs hollandais » du sud de Ceylan. Les chroniqueurs anglais traitèrent avec le plus grand mépris ces compatriotes européens en qui ils voyaient « une race de sang mêlé, aux mœurs peu communes $»^{22}$. Un siècle plus tard, lorsque Joseph Chailley-Bert tente de comprendre les circonstances sociales et politiques de la présence des métis à Java, il reprend tout en les critiquant les ambivalences et partis pris qui circulent dans certains secteurs de «l'opinion publique... [la] jalousie

20 Sur le personnage hybride du «métis », voir le récit classique qu'en fait Taylor 2009 [1983].

21 Maurik $1897: 140$.

22 Bosma \& Raben $2008: 82$. 
de ce que la loi concède aux métis, de tous ces emplois où on les admet. On leur impute toutes sortes de méfaits ou de mauvais desseins. On les taxe tantôt d'incapacité et tantôt de perfidie ». Au $\mathrm{XX}^{\mathrm{e}}$ siècle encore, la facilité apparente avec laquelle les métis des Indes néerlandaises se sont intégrés continue de choquer les visiteurs anglais. Comme le fait remarquer un anthropologue américain en 1942 à propos des Britanniques, « il leur était impossible d'envisager l'existence de sang-mêlé » ${ }^{23}$. Quant à la journaliste anglaise Harriet Winifred Ponder, qui cherche aussi à expliquer le phénomène dans les années 1930, elle constate simplement que les Hollandais sont « réalistes » : loin de traiter les Indo-Européens comme une minorité, ils ont au contraire la sagesse d'admettre que le métissage est une conséquence inévitable de la colonisation. En comparaison, elle trouve que le « mépris bien particulier » des Britanniques, qui refusent à la grande majorité des Anglo-Indiens l'accès à tous les lieux spécifiquement européens en Inde, témoigne de leur hypocrisie ainsi que d'une grossière erreur de jugement ${ }^{24}$.

Après tout, George Orwell n'a-t-il pas écrit dans son roman intitulé Une bistoire birmane (1934) que, sous le Raj britannique, les clubs de gentlemen implantés sur le continent indien formaient un réseau de "citadelle[s] spirituelle[s]» de défense de la masculinité britannique ? Ces clubs constituaient les seuls endroits où les Anglais pouvaient « se saouler ensemble, partager leurs repas et faire semblant d'être amis »; eux-mêmes parlaient d'une "nécessité politique à se fréquenter $»^{25}$. Mrinalini Sinha a récemment fait observer que les corps des blancs pauvres et des sabibs de couleur (les notables indigènes) étaient la preuve tangible qu'ils ne pouvaient prétendre postuler à l'entrée de ce cercle très fermé et très étroit constitué de vrais Anglais. Les signes d'authentique anglicité (Englishness) ne correspondaient pas de manière simple et automatique au nombre d'Européens vivant en Inde, qui s'élevait à environ 157000 personnes d'après le recensement de 1921, et dont près de la moitié étaient des "Eurasiens », comme le soulignait déjà le recensement de

\footnotetext{
23 Kennedy 1968 [1942] : 161.

24 Ponder 1990 [1942] : 153.

25 Orwell 1962 [1934] : 17, 38-39.
} 
1901. Pour autant, l'ostracisme dont faisaient l'objet ces Eurasiens, mais aussi les Anglais qui occupaient des postes subalternes ou les Indiens occidentalisés et éduqués des classes supérieures, était nécessairement «conçu comme un moyen de signaler une représentation spécifique et nettement délimitée de l'identité blanche (whiteness) en Inde $»^{26}$. Cette politique n'avait absolument rien de commun avec la situation dans l'empire colonial hollandais. De manière générale, on peut raisonnablement affirmer qu'au sein de la société coloniale hollandaise d'Asie du Sud-Est, et ce jusqu'à la fin du XIX ${ }^{e}$ siècle, l'autorité politique, l'influence économique et le prestige social n'étaient pas indexés sur la seule peau blanche des individus. $\mathrm{Au}$ contraire, pouvoir et statut résultaient d'une combinaison de facteurs aussi différents que « la classe, la profession exercée, l'origine géographique, la religion, le type de formation et la couleur de la peau; tous contribuaient à situer l'individu dans la hiérarchie sociale $»^{27}$.

\section{La modernité contestée de l'« apartheid » racial du XXe siècle ${ }^{28}$}

$\mathrm{Au}$ tournant du $\mathrm{XX}^{\mathrm{e}}$ siècle, des structures juridiques et des sensibilités politiques nouvelles émergent dans l'Indonésie coloniale. En 1899, la loi avait introduit un système tripartite de classification associant appartenance ethnique et citoyenneté : on distinguait les Européens (dont faisaient aussi partie des individus originaires du Japon, définis comme des Européens «honoraires»), des étrangers d'Orient (regroupant tant les Chinois que les Arabes) et les habitants de l'île (inlanders), c'est-à-dire la population indigène. Deux ans plus tard, la jeune reine Wilhelmina inaugure l'ère de la «Politique éthique », conçue pour moderniser l'infrastructure sociale et économique des Indes néerlandaises. Cette nouvelle politique se propose également d'améliorer la prise en charge sanitaire et l'éducation des populations locales, de façon à rembourser en quelque sorte une «dette d'honneur » contractée à l'égard d'un peuple dont le

\footnotetext{
26 Sinha $2005: 188$.

27 Bosma \& Raben $2008: 24$.

28 Cette notion controversée qu'est l'Apartheid, dans sa relation avec l'ordre racial du XXe siècle, a été utilisée pour la première fois par Tessel Pollmann en 1986.
} 
travail depuis la deuxième moitié du XIXe siècle avait permis aux PaysBas de réaliser des profits extraordinaires à Java et à Sumatra ${ }^{29}$. Ce tournant politique provoque l'arrivée d'un grand nombre d'immigrants en provenance de Hollande: professeurs, médecins, ingénieurs, fonctionnaires mieux formés, tous accompagnés de leur femme et de leurs enfants. On les appelle alors les totoks (littéralement «Hollandais de pure souche»et, plus communément, "nouveauxvenus ») ou les singkeh (qui désigne à l'origine "un immigré chinois soumis aux traditions", mais qui est ensuite utilisé pour faire référence aux nouveaux immigrés hollandais).

Cette réforme de l'ordre politique et moral est également motivée par l'augmentation des investissements nationaux et étrangers dans le fonctionnement capitaliste de l'archipel indonésien. Dans ce merveilleux nouveau monde (brave new world) qui s'impose au $\mathrm{XX}^{\mathrm{e}}$ siècle, des pays aussi puissants que les États-Unis, dont l'économie est largement dynamisée par une industrie automobile florissante (comme celle de Détroit), sont très intéressés par l'acquisition de précieuses nouvelles matières produites en Indonésie (caoutchouc, pétrole, bauxite). Ces demandes en provenance des États-Unis et de l'Europe occidentale s'ajoutent aux ambitions internationales d'exportation des matières premières depuis longtemps exploitées comme le tabac, le café, le thé, le sucre, la quinine. Grâce à une politique favorable à l'investissement étranger, l'activité de production et le marché de l'emploi du secteur privé connaissent un essor impressionnant. Ces nouveaux apports financiers représentent une véritable manne pour le développement économique, et attirent en Indonésie nombre de nouveaux colons venus de divers pays occidentaux: techniciens travaillant dans les puits de pétroles, ingénieurs spécialisés dans les mines et ingénieurs agronomes. Comme on pouvait s'y attendre, la plupart de ces immigrants blancs, d'origines nationales diverses, importent avec eux des conceptions des différences de classes et de couleurs qui entrent souvent en

29 Le travail le plus complet sur la question de la «politique éthique » est celui de Locher-Scholten 1981. 
conflit avec la mentalité et les habitudes de la communauté coloniale hollandaise, bigarrée et métissée ${ }^{30}$.

La concomitance de plusieurs facteurs (réorientation de la politique coloniale, montée en puissance du capitalisme et projet modernisateur) vient donc remettre en question, de manière volontaire et involontaire, les traditions de tolérance relative qui régnaient jusque-là vis-à-vis des mariages interraciaux et des nombreuses situations de concubinage. Le régime de mœurs que l'on voit surgir au tout début $\mathrm{du} \mathrm{XX}^{\mathrm{e}}$ siècle, qui cherche à rigidifier et à moderniser les frontières raciales internes à la société coloniale hollandaise, institue ainsi de nouvelles règles de fonctionnement des relations entre les sexes suivant l'appartenance ethnique ou sociale de chacun :

[...] sans que l'on puisse parler d'un régime d'apartheid au sens contemporain du mot, la société coloniale était [en train de devenir] un édifice bâti sur un grand partage racial inaugural, à partir duquel se déclinaient le jugement moral et l'estime sociale ${ }^{31}$.

Le fait d'être un "pur» Européen devient un réel signe distinctif et impose des définitions plus étroites et des identifications symboliques, surtout aux femmes de la communauté européenne. En d'autres termes, l'accès à la modernité exige que l'on se plie à de nouvelles normes du fait d'une configuration coloniale fondée sur une séparation plus stricte entre maîtres européens et sujets indigènes. C'est ce que l'on a pu décrire comme un phénomène d'occidentalisation ou de «totokisation des relations sociales [qui] accentue l'attachement des Européens citadins aux styles de vie de la mère patrie et plaide en faveur de l'importation de techniques de ségrégation normatives $»^{32}$.

$\mathrm{Au}$ début du $\mathrm{XX}^{\mathrm{e}}$ siècle, on voit donc ces nouveaux immigrants aux yeux bleus, à la peau blanche et venus d'Europe du Nord travailler de concert à la construction d'une solidarité intraeuropéenne, en collaboration parfois avec la frange aisée des Européens locaux, dont la couleur trahit les origines métissées. Ils

30 Fasseur $1992: 216-229$.

31 Bertrand 2005 : 594.

32 Bertrand 2005 : 598. 
associent femmes et enfants à ce processus, dans un effort surhumain (et voué à l'échec) pour créer l'unanimité au sein de l'enclave coloniale. Or, malgré toutes ces tentatives pour imposer de nouvelles valeurs et de nouvelles taxonomies raciales, les modèles anciens et éprouvés de «rapprochement et de mélange ethnique » perdurent jusqu'au déclenchement de la Seconde Guerre mondiale dans le Pacifique ${ }^{33}$. Comme l'écrit en 1928 le Consul général de Batavia Coert du Bois au Département d'État de Washington, DC :

Aux yeux de l'homme moyen, le physique de la jeune métisse est bien plus attirant que celui de la jeune Hollandaise de pure souche. Un tel constat est attesté par le nombre de mariages contractés par de jeunes Hollandais avec des métisses, au grand dam des éléments conservateurs de la société hollandaise ${ }^{34}$.

Parallèlement, la toute récente unanimité «imaginée» de la communauté européenne aux prises avec le « gothique tropical» de la société coloniale se met à céder devant l'incapacité des Hollandais-e-s à dépasser leurs propres préjugés de classes ${ }^{35}$.

En effet, dans les métropoles européennes, quand ils se retrouvent dans un dîner, au théâtre ou dans leurs clubs, les membres des classes supérieures et des classes moyennes ne sont guère amenés à croiser «les autres rangs » de la société ni même des personnes de couleur. La situation est bien différente dans les Indes néerlandaises, où de telles interactions sociales sont quotidiennes. Dans les grandes villes de l'île de Java, une Indo-Hollandaise couleur pain d'épices issue d'une riche famille de la région partage régulièrement la table de bridge ou le parquet de danse avec une matrone de l'aristocratie hollandaise, même si cette dernière la considère avec mépris du fait de

33 Bosma \& Raben 2008 : 343.

34 Administration chargée des archives nationales et dossiers nationaux, Coert du Bois, Consul Général des États-Unis à Batavia au Secrétaire d'État à Washington DC. Rapport volontaire III : «Le problème des métis », 9 octobre 1928, M-682, Dossiers du Département d'État relatif aux Affaires Internes des Pays-Bas, 1910 1929, Roll 33, 856D.00-.40. Stoler (2001) a analysé ce passage d'un autre point de vue. Voir www.historycooperative.org/journals/jah/88.3/stoler.html, consulté le 11 décembre 2010.

35 Les expressions «imaginées » et «gothique tropical » sont de Anderson 1983 : 137-139. 
leur différence d'origine et de statut. Une épouse de fonctionnaire de la classe moyenne, dotée d'un certain bagage universitaire et d'un esprit vif et imaginatif, peut être amenée à côtoyer l'épouse métisse et quasiment illettrée d'un officier de l'armée, parce qu'elle est la seule homologue « européenne » de cette région éloignée où son mari a été affecté. Dans la région des plantations de la côte Est de Sumatra, où la concurrence économique est particulièrement vive, il paraît normal qu'une femme d'origine patricienne s'incline devant une ancienne vendeuse d'Amsterdam ou devant une beauté indo-hollandaise du fait que leurs maris respectifs ont gravi plus vite les échelons de la hiérarchie d'une plantation de caoutchouc ou de tabac ${ }^{36}$. En 1910, l'ex-épouse d'un officier de l'armée hollandaise cantonné dans une rude région montagneuse de Sulawesi s'interroge en ces termes:

[...] pourquoi, dans les Indes, les femmes [Européennes] doivent-elles se contenter de la compagnie d'autres femmes «blanches » en fonction de leur rang (c'est un monde !), lequel est déterminé par le statut de leur époux ?37

C'est donc dans leur comportement quotidien que les épouses ainsi «intégrées » se voient confier une double responsabilité. Elles incarnent et policent une grande variété de distinctions hiérarchiques fondées sur des différences de couleurs et de classes. Elles deviennent alors les garantes de l'unité ontologique du système colonial, même si elles n'interviennent pour ainsi dire jamais dans le fonctionnement politique et économique de ce même système ${ }^{38}$. Elles endossent ainsi, avec plus ou moins d'entrain, le rôle symbolique de fantassins chargés de consolider et de défendre une hiérarchie coloniale qui place les femmes indigènes au bas de l'échelle et les Européens au sommet. Du fait de leur exclusion des fonctions officielles, tant politiques qu'économiques, les femmes blanches n'ont guère d'autre choix que de s'occuper de la maison, des enfants et des domestiques, et de soigner l'image de marque de leur famille, elle-même indexée sur la

36 Szekely-Lulof 1987 [1931] propose une description épouvantable de ce genre de confrontations.

37 Zeggelen 1989 [1910] : 135.

38 Pour une analyse de l'incorporation des épouses dans le système hiérarchique masculin, voir différents articles dans Callan \& Ardener 1984. 
fonction sociale de leur mari. Aux yeux de la gente masculine, l'obsession mesquine des femmes blanches pour le pedigree social prouve, de manière tautologique, leur incapacité naturelle à assumer le moindre rôle officiel. Ainsi voit-on fleurir des ouvrages sur la vie domestique incitant les mères à s'investir davantage dans l'éducation de leurs enfants au lieu de la déléguer à une nourrice indigène ( $b a b u)$. Les Européennes elles-mêmes, dont certaines ont une mère javanaise mais que leur père hollandais a reconnues, tandis que d'autres ont acquis un statut d'européennes par le mariage, ont plutôt tendance à s'accommoder des responsabilités symboliques qui leur incombent. Cette accentuation de la diversité des rangs et des statuts aux colonies vient en effet conforter chez les Européennes la conscience de leur supériorité vis-à-vis de leurs homologues indigènes, ne serait-ce que parce que la consolidation de ces clivages confère une signification sociale à ce qui relève de la sphère privée.

Inévitablement, un certain nombre de voix dissidentes s'élèvent contre ce nouvel ordre racial. Parmi elles, celle de l'écrivaine hollandaise Augusta de Wit, dont le désaccord transparaît dans son roman intitulé De godin die wacht (La Déesse qui attend, 1903). Elle y décrit la vie d'un groupe de familles hollandaises installées dans une région productrice de sucre à Java. On y voit des personnages qui se sont attribué le noble titre d'Européens dans le seul but de s'élever au-dessus de la population javanaise qu'ils considèrent comme «des créatures moitié animales, moitié idylliques, à mi-chemin entre le berger buriné peint par Watteau et l'orang-outang »39. De Wit brosse là une caricature féroce des magnas hollandais conservateurs qui règnent sur le marché du sucre, mais aussi de leurs abominables épouses, tous persuadés que seuls leurs efforts et leur travail ont transformé Java en une terre de «lait, plus précisément de lait de coco, et de miel »- raison pour laquelle ils s'octroient la liberté de se comporter comme si cette terre et ses habitants leur appartenaient ${ }^{40}$. Cette tendance croissante à valoriser le lien entre culture et identité occidentales d'un côté, et origines européennes et fantasme de la peau blanche de l'autre a pour objectif de remettre en cause, par des biais

\footnotetext{
39 De Wit $1903: 39-40$.

$40 \quad$ De Wit 1903 : 39.
} 
plus ou moins assumés, une pratique communément acceptée, consistant à inclure des citoyens d'origines raciales mixtes dans le corps politique des Indes néerlandaises. Si ce nouveau régime d'occidentalisation ou de "totokisation » ne rencontre qu'un succès mitigé, il provoque un désarroi certain parmi ceux qui sont originaires de la région, et encourage des antagonismes politiques totalement inédits au sein de la communauté coloniale hollandaise. Ainsi, le journaliste Willem Walraven raconte que, dans les années 1920 à Java, sa fille aînée, qui avait une peau relativement pâle, ne voulait pas être vue en public avec sa mère, une Sundanaise à la peau sombre de l'ouest de Java, de peur que ses camarades d'école européens ne se moquent d'elle et ne la rejettent. Walraven s'est rendu célèbre pour le mépris que lui inspirait l'hypocrisie de la communauté coloniale hollandaise, et tout particulièrement ses commères, mesquines et bourrées de préjugés. Après la naissance de sa première fille en 1919, il avait été scandalisé par les propos d'une soi-disant grande dame hollandaise: celle-ci avait mis en garde sa future épouse, lui expliquant qu'en tant que père hollandais digne de ce nom, Walraven lui retirerait probablement la garde de l'enfant afin de protéger la fillette «de l'influence indigène insidieuse [de sa mère], de manière à l'élever à l'européenne. » Dans une lettre adressée à sa famille hollandaise, Walraven fulmine contre

la bassesse de cette bande d'Européens dont la sophistication est une pure farce, et parmi lesquels se trouvent des escrocs notoires, dont les épouses ne sont que de banales mégères...

Quant à sa fiancée sundanaise, constatant avec honte la différence de couleur entre elle et sa fille, elle refusait qu'on la photographie avec l'enfant dans les bras ${ }^{41}$.

\section{«Les femelles de son espèce} sont plus meurtrières que les mâles " ${ }^{42}$

Après 1900, la présence concrète et la visibilité sociale croissante de femmes blanches en provenance des Pays-Bas ou d'autres nations

\footnotetext{
41 Walraven 1992 : 384. Voir aussi Okker 2000 : 94-95.

42 Cité par Burton 1994 : 15.
} 
européennes participe d'un ensemble d'initiatives destinées à faire des Indes néerlandaises jusque-là imprégnées de culture métisse une enclave européenne fondée sur les différences de classes et de couleurs. Le hasard fait qu'un grand nombre de blanches émigrent en même temps, si bien que certains contemporains laissent entendre qu'elles sont responsables de cette toute nouvelle conception d'un apartheid «moderne », bien plus que les changements structuraux liés à un développement économique rapide, à des investissements croissants de capitaux ou même aux évolutions politiques locales. Cette thèse est étayée par le constat de l'ignorance des traditions et de la culture indigènes par les Néerlandaises. Une fois installées, ces femmes ont pu concevoir les Indes néerlandaises ou britanniques comme un univers inconnu et, de ce fait, effrayant, qui allait façonner leur quotidien et leur identité profonde. Ce nouvel environnement exotique a pu susciter un certain nombre d'angoisses vis-à-vis du danger incarné par ces "autres » indigènes dans un monde étranger où ils vivent collectivement. En Inde britannique, au XXe siècle, de nombreuses émigrées anglaises fraîchement débarquées ne parviennent pas à surmonter le choc de la pauvreté et de la misère locales, de même qu'elles sont indignées par le sort réservé à «la femme indienne », contrainte de se soumettre à la pratique du suttee (immolation des veuves) ou du purdah (réclusion des femmes). La plupart d'entre elles se retranchent derrière un mur de dédain raciste et trouvent refuge dans la tour d'ivoire des lieux de sociabilité britanniques. Quelques-uns de leurs contemporains leur reprochent de venir bouleverser la coopération fraternelle et harmonieuse qui, d'après eux, régnait entre hommes blancs et hommes de couleur avant qu'elles n'arrivent ${ }^{43}$. Le prolifique Rudyard Kipling accrédite de telles assertions quand il écrit dans son célèbre poème intitulé « La Femelle de l'espèce » (1911) :

And Man knows it! Knows, moreover, that the Woman that God gave him Must command but may not govern - shall enthrall but not enslave him. And She knows, because She warns him, and Her instincts never fail, That the Female of Her Species is more deadly than the Male ${ }^{44}$

\footnotetext{
43 Teo 2004. S'agissant des Indes néerlandaises, voir Buchheim 2009.

44 www.heretical.com/miscella/kipling1.html. Consulté le 16 décembre 2010.
} 
Et l'homme le sait! Il sait, en outre, que la Femme que Dieu lui a donnée Doit commander, mais ne peut pas gouverner - doit le fasciner mais pas l'asservir.

Et Elle le sait, car Elle le met en garde, et Ses instincts ne la trompent jamais, La Femelle de son espèce est plus meurtrière que le mâle.

L'essayiste et journaliste Rudy Kousbroek, originaire des Indes néerlandaises, propose une analyse semblable. Il dresse un portrait caricatural des Hollandaises dont les idées toutes faites sur la population indigène et dont le mépris vis-à-vis des nombreux métis de la communauté européenne auraient contribué, en ce début de $\mathrm{XX}^{\mathrm{e}}$ siècle, à forger une société fondée sur des distinctions raciales plus fortes. À propos des femmes blanches, il écrit qu'elles sont

de poignantes créatures victoriennes mises sur un piédestal, nées pour souffrir, à peine capables de se prendre en charge..., insensibles aux problèmes environnants et dépourvues de toute libido.

Curieusement, dans la même page, Kousbroek présente l'archétype de la matrone hollandaise des Indes (mem) comme une «coquette vaniteuse et cruelle» dont le point de vue est bien plus colonial et raciste que celui de son mari; il poursuit en disant que, dans l'ensemble, la plupart de ces mems «naïves et hypocrites ne sont bonnes qu'à propager les ragots $»^{45}$.

Ce genre d'hyperboles, toutefois, est révélateur des préjugés masculins sur le comportement des femmes, même s'il illustre le rôle symbolique attribué à celles qui se devaient d'incarner ladite supériorité culturelle de la communauté coloniale hollandaise. De fait, aucune Européenne ni Indo-Hollandaise n'avait de responsabilités politiques ou économiques, bien que certaines d'entre elles fussent de riches héritières. Aucune femme ne participait aux activités classiques du gouvernement, du commerce ou de la production, activités qui impliquaient de flatter les notables locaux, de surveiller les employés indo-hollandais ou encore d'infliger des châtiments corporels à des coolies javanais ou chinois travaillant dans les plantations de tabac ou de caoutchouc. On peut donc dire que les colons se servaient de leurs femmes et de leurs filles pour les épauler dans la consolidation de la dignité morale et de la hiérarchie interne à la communauté

45 Kousbroek $1992: 356$. 
européenne mais que, dans le même temps, ils étaient prompts à les rendre responsables de la moindre déchéance de leur statut. Au XX siècle, la conduite personnelle de ces femmes vivant aux Indes néerlandaises était bel et bien investie d'une signification publique particulière du fait qu'elles contribuaient au maintien de la prééminence morale et de la mythique blancheur de la communauté européenne.

\section{Quelques remarques pour conclure}

Malgré le discours récurrent au début du $\mathrm{XX}^{\mathrm{e}}$ siècle, qui incite à respecter l'étanchéité des frontières raciales au sein de la société des Indes néerlandaises, de nombreux Européens continuent à épouser des femmes indigènes ou métisses sans impunité aucune. En 1925, $27,5 \%$ des Européens qui contractent un mariage choisissent une compagne indigène ou métisse ; cette proportion est encore de $20 \%$ en 194046. Au cours des années 1930, 30\% seulement de la population officiellement recensée comme européenne est véritablement originaire des Pays-Bas ou d'un autre pays occidental. Les 70\% d'Européens restants font partie de la population créolisée et ont tendance à penser que les Pays-Bas se trouvent sur une autre planète, voire dans une autre galaxie. Le sociologue Wim Wertheim estime ainsi qu'en 1942, en Indonésie, 8 à 9 millions d'habitants (sur une population totale d'environ 50 millions) sont vraisemblablement métis ${ }^{47}$.

L'idée et la réalité même d'une culture syncrétique propre aux Indes néerlandaises se maintiennent jusqu'à l'occupation japonaise en 1942 et ne trouvent pas d'équivalent aux Philippines américaines, ni dans les Empires britannique ou français de l'Asie du Sud-Est. Le commentaire de Joseph Chailley-Bert, qui explique qu'en 1900 les habitants des colonies hollandaises «ont dépouillé le vieil homme européen et sont entrés, quelques-uns dans la peau, mais tous dans le régime du Javanais ", est encore pertinent en 194248. Aussi superficielles qu'aient pu être leurs intuitions, il est évident que, dans

\footnotetext{
46 Fasseur 1992: 223.

47 Cité par Harms \& Pollmann 1992 : 11.

48 Chailley-Bert $1900: 72$.
} 
l'Indonésie coloniale, la plupart des Européen-ne-s avaient une certaine connaissance des coutumes et traditions (adat) du pays, des variantes locales de l'islam et d'autres religions, du théâtre d'ombres, de la musique de gamelan, des plantes médicinales et des croyances populaires dans les pouvoirs magiques de la nature tropicale au sein de laquelle ils vivaient collectivement. Les hommes européens, indépendamment de leur statut social en Europe, s'associaient à d'autres hommes, nés dans l'archipel indonésien et dont la peau était en général plus sombre, pour mieux se dissimuler derrière un imaginaire "rideau de blancheur impénétrable», comme l'écrit si justement la romancière afro-américaine Toni Morrison ${ }^{49}$. Si l'identité raciale des femmes blanches, tout comme celle des femmes indigènes, était essentiellement mouvante $\mathrm{du}$ fait de la fluctuation des classifications légales liées aux mariages et aux reconnaissances en paternité, l'identité européenne des hommes était, elle, presque toujours immuable ${ }^{50}$. Dans les Indes néerlandaises, la prétendue blancheur de la communauté européenne est un mythe construit de toutes pièces sur l'illusion d'une indifférence tant à l'appartenance sociale qu'à la couleur de la peau, et elle sert surtout de marqueur de masculinité. L'exemple de cette situation coloniale permet également de souligner que les régimes raciaux ne s'ancrent pas dans «une idéologie a priori», comme Emmanuelle Saada l'a montré, "mais [dans une idéologie qui] se construit dans des rapports de pouvoir» au sein d'une zone de contact colonial spécifique ${ }^{51}$. Ainsi, la surveillance de la conduite personnelle des femmes et de leur aptitude à procréer est-elle un moyen de s'assurer, autant que possible, de leur participation à l'accumulation des immenses profits financiers et matériels générés par la colonisation.

Article traduit par Florence Cabaret, ERIAC, EA 4307, Université de Rouen.

\footnotetext{
49 Morrison $1992: 32-33$.

50 L'exception concerne les Staatsblad Europeanen, c'est-à-dire les membres de l'élite indigène qui bénéficient de dispenses et sont reconnus comme européens.

51 Saada 2006.
} 


\section{Bibliographie}

ANDERSON Benedict, 1983, Imagined Communities: Reflections on the Origin and Spread of Nationalism, London/New York, Verso.

BAAY Reggie, 2008, De njai. Het concubinaat in Nederlands-Indië, Amsterdam, Athenaeum - Polak \& Van Gennep.

BERTRAND Romain, 2005, État colonial, noblesse et nationalisme à Java, Paris, Karthala.

Bosma Ulbe \& Remco Raben, 2008, Being 'Dutch' in the Indies: A History of Creolisation and Empire, 1500-1920, Athens, Ohio University Press.

BuCHHEIM Eveline, 2009, «Passie en missie. Huwelijken van Europeanen in Nederlands-Indië en Indonesië, 1920-1958 », University of Amsterdam, Ph.D. dissertation.

BurTON Antoinette, 1994, Burdens of History: British Feminists, Indian Women and Imperial Culture, 1865-1915, Chapel Hill, University of North Carolina Press.

Callan Hillary \& Shirley ARDEnER (eds), 1984, The Incorporated Wife, London, Croom Helm.

Chailley-Bert Joseph, 1900, Java et ses habitants, Paris, Armand Colin.

-, 1891-1892, Revue des deux mondes, 108/109/110.

Clancy-Smith Julia \& Frances Gouda (eds), 1998, Domesticating the Empire: Race, Gender and Family Life in French and Dutch Colonialism, Charlottesville, The University Press of Virginia.

Davidoff Leonore \& Catherine Hald, 1987, Family Fortunes: Men and Women of the English Middle Class, 1780-1850, Chicago, University of Chicago Press.

DE WiT Augusta, 1903, De godin die wacht, Amsterdam, Van Kempen.

FASSEUR Cees, 1980, Geld en geweten. Een bundel opstellen over anderbalve eeuw Nederlands bestuur in de Indonesische archipel, The Hague, Martinus Nijhoff Press.

-, 1992, "Hoeksteen en struikelblok. Rassenonderscheid en overheidsbeleid in Nederlands-Indie », Tijdschrift voor Geschiedenis, 105, 2, p. 216-229.

GoudA Frances, 2008 [1995], Dutch Culture Overseas: Colonial Practice in the NetherlandsIndies, 1900-1942, Jakarta, Equinox Publishers.

Harms Ingrid \& Tessel Pollmann, 1992, "In Nederland door omstandigheden », Bijvoegsel V rij Nederland, 17, May 15.

KEAY John, 1997, Empire's End. A History of the Far East from High Colonialism to Hong Kong, New York, W.W. Norton.

KenneDy Raymond, 1968 [1942], The Ageless Indies, , New York, Greenwood Press. 
KNAPMAN Gareth, 2008, «Race, Empire and Liberalism: An Interpretation of John Crawfurd's History of the Indian Archipelago », www.arts.monash.edu.au/mai/asaa/garethknapman.pdf

Kousbroek Rudy, 1992, Het Oostindische kampsyndroom, Amsterdam, Meulenhoff.

LOCHER-SCHOLTEN Elsbeth, 1981, Ethiek in fragmenten. Vijf studiën over koloniaal denken en doen van Nederlanders in de Indonesische archipel, 1877-1942, Utrecht, HES Uitgevers.

Marshall Peter J., 1992, "British Immigration into India in the Nineteenth Century », in P.C. EMMER \& M. MÖRNER (eds), European Expansion and Migration: Essays on the Intercontinental Migration from Africa, Asia, and Europe, New York/Oxford, Berg Publishers, p. 179-196.

MAURIK Justus van, 1897, Indrukken van een totok. Indische typen en schetsen, Amsterdam, van Holkema \& Warendorf.

MORrison Toni, 1992, Playing in the Dark. Whiteness and the Literary Imagination, Cambridge, Harvard University Press.

OKKER Frank, 2000, Dirksland tussen de doerians. Een biografie van Willem Walraven, Amsterdam, Uitgeverij Bas Lubberhuizen.

Orwell George, 1962 [1934], Burmese Days, New York/London, Harcourt Harvest Books.

Pollmann Tessel, 1991, «Bruidstraantjes. De koloniale roman, de njai en de apartheid ", in Jaarboek voor V rouwengeschiedenis $\mathrm{n}^{\circ} 7$, reproduit dans Bruidstraantjes en andere geschiedenissen, The Hague, Sdu Uitgeverij, p. 9-31.

Pomfret David M., 2009, «Raising Eurasia: Race, Class and Age in French and British Colonies ", Comparative Studies in Society and History, 51, p. 314-343.

Ponder H.W., 1990 [1942], Javanese Panorama. More Impressions of the 1930's, Singapore, Oxford University Press.

SAADA Emmanuelle, 2003, "Citoyens et sujets de l'empire français. Les usages du droit en situation coloniale », Genèses, 53, p. 4-24.

-, 2006, compte rendu d'Olivier le Cour Grandmaison, Coloniser, exterminer : sur la guerre et l'État colonial, Critique internationale, 32, p. 2-3.

-, 2007, Les Enfants de la colonie: les métis de l'empire français entre sujétion et citoyenneté, Paris, La Découverte.

SINHA Mrinalini, 2005, "Britishness, Clubbability and the Colonial Public Sphere », in Tony Ballantyne \& Antoinette Burton (eds), Bodies in Contact. Gender and the Body in World History, Durham, Duke University Press, p. 184-200. 
Stoler Ann Laura, 1992, "Sexual Affronts and Racial Frontiers: European Identities and the Cultural Politics of Exclusions in Colonial Southeast Asia », Comparative Studies in Society and History, 34, p. 514-551.

-, 2001, «Tense and Tender Ties: The Politics of Comparison in North American History and (Post) Colonial Studies ", Journal of American History, 88/3, p. 829-865.

SzEKELY-Lulof Madelon, 1987 [1931], Rubber, Singapore, Oxford University Press.

TAYLOR Jean Gelman, 2009 [1983], The Social World of Batavia: Europeans and Eurasians in Colonial Indonesia, Madison, University of Wisconsin Press.

TeO Hsu-Ming, 2004, "Romancing the Raj: Interracial Relations in Anglo-Indian Romance Novels », History of Intellectual Culture, 4/1, p. 1-18.

WALRAVEN Willem, 1992, Brieven aan familie en vrienden, 1919-1941, Amsterdam, Van Oorschot.

ZEGGELEN Marie van, 1989 [1910], Indrukken van een zwervelinge. De Hollandse vrouw in Indie, Schoorl, Conserve. 